\title{
Validation of the Chinese version of Brief Assessment of Cognition in Schizophrenia
}

This article was published in the following Dove Press journal:

Neuropsychiatric Disease and Treatment

31 October 2016

Number of times this article has been viewed

\author{
Liang-Jen Wang' \\ Pao-Yen Lin ${ }^{2,3}$ \\ Yu Lee ${ }^{2}$ \\ Yu-Chi Huang ${ }^{2}$ \\ $\mathrm{Su}$-Ting $\mathrm{Hsu}^{4}$ \\ Chi-Fa Hung ${ }^{2}$ \\ Chih-Ken Chen ${ }^{5,6}$ \\ Yi-Chih Chen ${ }^{5,6}$ \\ Ya-Ling Wang ${ }^{2}$ \\ Ming-Che Tsai ${ }^{2}$
}

'Department of Child and Adolescent Psychiatry, Kaohsiung Chang Gung Memorial Hospital and Chang Gung University College of Medicine, ${ }^{2}$ Department of Psychiatry, Kaohsiung Chang Gung Memorial Hospital and Chang Gung University College of Medicine, ${ }^{3}$ Institute for Translational Research in Biomedical Sciences, Kaohsiung Chang Gung Memorial Hospital, ${ }^{4}$ Department of Community Psychiatry, Kaohsiung Municipal KaiSyuan Psychiatric Hospital, Kaohsiung, ${ }^{5}$ Department of Psychiatry, Chang Gung Memorial Hospital, Keelung, ${ }^{6}$ Chang Gung University College of Medicine, Taoyuan, Taiwan, Republic of China
Correspondence: Liang-Jen Wang Department of Child and Adolescent Psychiatry, Kaohsiung Chang Gung Memorial Hospital, No 123, Ta-Pei Road, Niao-Song District, Kaohsiung City 833,

Taiwan, Republic of China

Tel +88677317123 ext 8753

Fax +88677326817

Email wangliangjen@gmail.com
Objective: A test battery that measures cognitive function impairment in patients with schizophrenia, the Brief Assessment of Cognition in Schizophrenia (BACS), has been translated into various languages and validated. This study aimed to test the reliability and validity of the Chinese version of the BACS in a Chinese-speaking population.

Methods: All participants in this study (66 patients with schizophrenia [mean age: 41.2 years, $57.6 \%$ male] and 66 age- and sex-matched healthy controls) were from Taiwan and assessed using the BACS and the University of California, San Diego (UCSD) Performance-Based Skills Assessment, Brief Version (UPSA-B). Thirty-eight of the 66 patients with schizophrenia received a reassessment using the BACS.

Results: The BACS had good test-retest reliability, and all BACS subtests had statistically insignificant practice effects. Principal components analysis demonstrated that a one-factor solution best fits our dataset ( $60.9 \%$ of the variance). In both patients and controls, the BACS composite scores were positively correlated with all BACS subscales $(P<0.001)$ and UPSA-B scales $(P<0.001)$. Furthermore, all BACS subtests (verbal memory, working memory, motor speed, verbal fluency, attention and processing speed, and executive function) significantly differentiated patients with schizophrenia from healthy controls $(P<0.001)$, and the BACS composite score had the best discriminative validity $(P<0.001)$.

Conclusion: The Chinese version of the BACS exhibits satisfactory psychometric properties, including high test-retest reliability, high internal consistency, acceptable concurrent validity, and good discriminant validity. We suggest that the BACS is a reliable and practical tool for assessing cognitive function in patients with schizophrenia.

Keywords: psychometrics, reliability, validity, cognitive function, schizophrenia

\section{Introduction}

Patients with schizophrenia exhibit a wide range of cognitive dysfunctions, ${ }^{1}$ including deficits in working memory, verbal and spatial memory, motor and processing speed, attention, and executive function. ${ }^{2,3}$ Cognitive impairment determines the level of patients' daily function and social-occupational outcome. ${ }^{4,5}$ Therefore, a cognitive test battery that can be efficiently and feasibly used to assess cognitive deficits in patients with schizophrenia is essential for outcome measurement in both clinical settings and research environments. ${ }^{6,7}$ The ideal test battery should contain a broad range of cognitive dimensions, satisfy reliability and validity criteria, and be repeatable to assess changes over long-term follow-up periods. ${ }^{8}$

Numerous neuropsychological testing batteries have been developed to determine the magnitude of cognitive impairment in patients with schizophrenia. ${ }^{9,10}$ For example, the well-known Measurement and Treatment Research to Improve Cognition in Schizophrenia is used in clinical trials for assessing cognitive function in patients with 
schizophrenia. ${ }^{11,12}$ However, most of the present cognitive assessment tools are time-consuming, costly, or inconvenient to use in practice. The Brief Assessment of Cognition in Schizophrenia (BACS) is a brief test battery which measures impairment of verbal memory, working memory, motor speed, verbal fluency, attention and processing speed, and executive function. ${ }^{13}$ These domains of cognitive deficits have been demonstrated to reliably reflect the functionality of patients with schizophrenia. ${ }^{14,15}$ The BACS is easily performed in clinical settings and can be administered by medical professionals in 40 minutes. The original version of BACS was in English and has undergone a validation study. ${ }^{16}$ The BACS has been translated into over 30 languages, and the validities of the Japanese, ${ }^{17}$ French, ${ }^{18}$ Italian, ${ }^{19}$ Spanish,${ }^{20}$ German, ${ }^{21}$ Persian, ${ }^{22}$ and Brazilian Portuguese ${ }^{23}$ versions have been proven.

The BACS has been adapted into Chinese version by the original authors. The normative data of the Chinese version of the BACS have been established using a representative community sample in Taiwan (unpublished data), and notable differences in verbal tests and executive function were found when compared to the norms developed in the US. ${ }^{13}$ However, the BACS has not been validated in a Chinese-speaking population. Since Chinese is one of the most commonly spoken languages in the world, adaptation and validation of the BACS could advance the knowledge about cognition in schizophrenia, facilitate the comparison of patients with schizophrenia in various countries, and help to determine the cross-cultural validity of the BACS. Therefore, the current study aimed to validate the BACS by comparing 66 patients with schizophrenia to an age- and sex-matched sample of healthy controls.

\section{Methods}

\section{Study participants}

This cross-sectional study was conducted in Chang Gung Memorial Hospital, and was approved by the Institutional Review Board of Chang Gung Hospital in Taiwan. A written informed consent was obtained from all the participants. Eligible patients with schizophrenia at an outpatient department in the Kaohsiung Chang Gung Memorial Hospital were selected for this study if they met the following inclusion criteria: 1) were aged 18-65 years; 2) were diagnosed with schizophrenia as defined by the Diagnostic and Statistical Manual of Mental Disorders, Fourth Edition $(D S M-I V-T R)^{24}$ after assessment using the Chinese version of the Mini International Neuropsychiatric Interview (MINI) (which was translated from English by the Taiwan Society of Psychiatry ${ }^{25}$ and known to have good inter-rater reliability); ${ }^{26}$ 3 ) had received a stable dose of an antipsychotic drug and had relatively stable psychotic symptoms; 4) had no history of major physical illnesses that would influence cognitive performance, and 5) could speak and read Chinese to perform the test. In total, 66 patients with schizophrenia were recruited in this study (mean age: 41.2 years, $57.6 \%$ male). Information regarding the antipsychotic usage by patients and daily dose were obtained through patients' medical records. The dose of antipsychotic medication in use was calculated according to the defined daily dose (DDD) recommended by the WHO Collaborating Centre for Drug Statistics Methodology. ${ }^{27}$

The healthy individuals were recruited from the staff of Kaohsiung Chang Gung Memorial Hospital and Keelung Chang Gung Memorial Hospital, and from the community volunteers in Kaohsiung City and Keelung City, Taiwan. The recruitment criteria were as follows: 1) absence of a history of major psychiatric disorders (eg, psychotic disorders, bipolar disorders, major depressive disorders, organic mental disorders, and substance user disorders) confirmed using $D S M-I V$ criteria and the MINI; 2) absence of any known systemic or neurological diseases that would influence cognitive performance; 3) Han Chinese ethnicity; and 4) capacity to speak and read Chinese to perform the test. We recruited 382 healthy individuals to establish the norms of the BACS (unpublished data). Finally, 66 healthy individuals who were age- and sex-matched to our patients with schizophrenia were selected as the control group.

\section{Cognitive assessment}

\section{The BACS}

The cognitive functions of all participants were assessed using the BACS. A total of 38 patients with schizophrenia were willing to receive BACS retesting, and the rest 28 patients either dropped out or refused to receive the retest. The BACS test battery is used for neuropsychological assessment of patients with various psychotic disorders, ${ }^{26}$ and the subtests of the BACS are listed in the following section.

List learning test (verbal memory): patients are presented with 15 phrases and then asked to recall as many as possible. This procedure is repeated five times. The outcome measure is the total number of phrases recalled. Eight alternative forms are designed to minimize the practice effect. In the current study, the first form was chosen for testing all participants, and the second form was chosen for retest purposes.

Digit sequencing task (working memory): patients are presented with clusters of numbers of increasing length and are asked to repeat the numbers in order, from lowest to highest. The trials are of increasing difficulty. The outcome measure is the total number of correct items.

Token motor task (motor speed): patients are given 100 plastic tokens and asked to place them into a container as 
quickly as possible for 60 seconds. The outcome measure is the total number of tokens placed in the container.

Verbal fluency: performance is evaluated based on the total number of words/phrases adequately responded to in the Category Instances Test and Controlled Oral Word Association Test. In the Category Instances Test (semantic fluency), patients are given 60 seconds to list as many words as possible relating to the category of animals. The outcome measure is the total number of unique words listed for animals. In the Controlled Oral Word Association Test (letter fluency), patients are given 60 seconds to list as many "phrases" as possible that begin with the Mandarin word "Kou" and "Jing" in two separate trials. The outcome measure is the total number of unique phrases listed.

Symbol coding test (attention and processing speed): as quickly as possible, patients are asked to write numerals 1-9 that matched the symbols on a response sheet for 90 seconds. The outcome measure is the total number of correct responses.

The Tower of London test (executive function): patients are asked to look at two pictures simultaneously. Each picture shows three differently colored balls arranged on three pegs, with the balls in a unique arrangement in each picture. The patients are asked to calculate the smallest number of times that the balls in one picture would have to be moved to arrive at an arrangement of balls as seen in another picture. The outcome measure is the number of trials needed to find the correct solution. There are two alternative forms. In the current study, the first form was used for testing all participants, and the second form was used for retesting.

Finally, a composite score was calculated by summing scores on each measure for each patient and healthy control. The $Z$-score compared the sum of each patient with that of the corresponding control. ${ }^{13}$ A $Z$-score of 0 for each scale indicates average functioning with reference to the normal population of the same age range and sex, and every 1 point represents one standard deviation (SD).

\section{The UCSD Performance-Based Skills Assessment, Brief Version (UPSA-B)}

The UPSA-B is modified from the UCSD Performance-Based Skills Assessment (UPSA) which is designed for assessing daily functioning in patients with severe mental illness. ${ }^{28}$ The UPSA-B consists of two subscales: a financial portion and communication domain. In the financial portion, the participant would be asked to count specific amounts of real currency, make change, and request a bank check to pay a bill. The communication domain requires the participant to correctly call directory assistance to obtain a telephone number and then reschedule an appointment in a hospital. The raw scores of the UBSA-B were standardized to a 100-point scale. ${ }^{29}$ The Chinese version of the UPSA-B was developed and has been validated in patients with mental illnesses among Chinese populations. ${ }^{30}$ The UPSA-B served as the validity criterion for the BACS in this study.

\section{Statistical analyses}

Data were analyzed using the statistical software package SPSS, version 21.0 (SPSS Inc., Chicago, IL, USA). The data are presented as either mean $( \pm \mathrm{SD})$ or frequency $(\%)$. Categorical variables were compared between patients and controls using either the chi-square test or Fisher's exact test, depending on case numbers, and an independent $t$-test was used to compare continuous variables between groups. In a two-tailed test, $P<0.05$ was considered statistically significant.

The test-retest reliability of all subtests of the BACS was measured with intraclass correlations (ICC). The paired samples $t$-test was used to examine whether there were significant practice effects. The principal components analysis (PCA) with varimax rotation was applied for determining the factor structure of the BACS subtests. Pearson's correlation was performed to analyze the relationships between each BACS subscale and the correlation between BACS composite scores and UPSA-B scale scores, separately in patients and controls.

Scaled Z-scores for a subset of variables and a composite $Z$-score were calculated based on the normative data of the BACS established in our previous work (unpublished data). The receiver operating characteristic (ROC) analyses and area under curve (AUC) were further applied to examine the validity of using the $Z$-score of each BACS subtest to differentiate patients with schizophrenia from healthy controls. Sensitivity and specificity of the best cutoff point of the BACS composite score were calculated.

A Kruskal-Wallis test was performed to examine the potential difference in BACS composite scores among schizophrenia patients with different antipsychotic use. Pearson's correlation test was performed to analyze the correlation between DDD of antipsychotics and BACS composite scores.

\section{Results}

A total of 66 patients with schizophrenia (schizophrenia group) and 66 age- and sex-matched healthy control subjects (control group) were recruited in this study. The demographic characteristics and BACS performance of the groups are listed in Table 1. A higher proportion of the control 
Table I Demographic characteristics of patients with schizophrenia and healthy control subjects

\begin{tabular}{|c|c|c|c|c|c|c|}
\hline \multirow[t]{2}{*}{ Variables } & \multicolumn{2}{|c|}{ Patients $(\mathrm{N}=66)$} & \multicolumn{2}{|c|}{ Controls $(\mathrm{N}=66)$} & \multicolumn{2}{|c|}{ Statistical value } \\
\hline & $\mathbf{N}$ or mean & $\%$ or SD & $\mathbf{N}$ or mean & $\%$ or SD & $x^{2}$ or $t$ & $P$-value \\
\hline Age (years) & 41.24 & 10.06 & 41.24 & 10.09 & 0.000 & 1.000 \\
\hline Sex & - & - & - & - & 0.000 & 1.000 \\
\hline Male & 38 & 57.6 & 38 & 57.6 & - & - \\
\hline Female & 28 & 42.4 & 28 & 42.4 & - & - \\
\hline Marital status & - & - & - & - & 60.045 & $<0.001 *$ \\
\hline Married & 6 & 9.1 & 50 & 75.8 & - & - \\
\hline Not married & 60 & 90.9 & 16 & 24.2 & - & - \\
\hline Occupation & - & - & - & - & 46.964 & $<0.00 I^{*}$ \\
\hline Employed & 18 & 27.3 & 57 & 86.4 & - & - \\
\hline Unemployed & 48 & 72.7 & 9 & 13.6 & - & - \\
\hline Education (years) & 12.50 & 3.26 & 14.88 & 2.68 & -4.654 & $<0.00 I^{*}$ \\
\hline BACS performance & - & - & - & - & - & - \\
\hline Verbal memory & 26.21 & 11.09 & 41.36 & 9.50 & -8.430 & $<0.00 I^{*}$ \\
\hline Working memory & 15.62 & 4.59 & 23.17 & 9.70 & -5.712 & $<0.00 I^{*}$ \\
\hline Motor speed & 57.27 & 13.13 & 81.38 & 12.52 & -10.754 & $<0.00 I^{*}$ \\
\hline Verbal fluency & 23.97 & 7.41 & 34.35 & 7.68 & -7.903 & $<0.00 I^{*}$ \\
\hline Attention and processing speed & 38.45 & 13.53 & 61.35 & 12.67 & -10.037 & $<0.00 I^{*}$ \\
\hline Executive function & 12.32 & 5.85 & 17.05 & 3.37 & -5.688 & $<0.00 I^{*}$ \\
\hline Composite score (Z-score) & -1.94 & 1.10 & 0.02 & 0.72 & -12.088 & $<0.00 I^{*}$ \\
\hline
\end{tabular}

Note: $* P<0.001$.

Abbreviations: BACS, Brief Assessment of Cognition in Schizophrenia; SD, standard deviation.

group were married $(P<0.001)$ and employed $(P<0.001)$. Compared with the control group, patients in the schizophrenia group were impaired in all dimensions of cognitive function assessed by the BACS and UPSA-B $(P<0.001)$.

The test-retest reliability and potential practice effects of the BACS are summarized in Table 2. In the 38 patients with schizophrenia who were tested and retested, the ICCs of the BACS subtests ranged from 0.59 (verbal fluency) to 0.86 (attention and processing speed), and all were significant $(P<0.001)$. The paired samples $t$-test revealed that none of the BACS subtests showed statistically significant practice effects.

PCA demonstrated that the first and second components accounted for $60.9 \%$ (eigenvalue: 3.66 ) and $14.9 \%$ (eigenvalue: 0.90 ) of the variance, respectively. This suggests that a one-factor solution is the best fit for our dataset.
Table 3 shows the correlation matrix of each BACS subtest of patients and controls. In patients with schizophrenia, the BACS composite score was positively correlated with all BACS subtest scores $(P<0.001)$, and all BACS subtest scores were positively correlated with each other $(P<0.05)$, with the exception of the correlation between working memory and motor speed. The composite score was significantly correlated with all subtest scores in controls $(P<0.01)$ and with UPSA-B scores (Figure 1) in patients $(r=0.70, P<0.001)$ and controls $(r=0.44, P<0.001)$.

The ROC analysis (Figure 2) showed that all BACS subtest scores could significantly differentiate patients with schizophrenia from healthy controls $(P<0.001)$. The BACS composite score had the best discriminant validity (AUC: 0.94, $P<0.001$ ), followed by the scores for motor speed (AUC: $0.91, P<0.001$ ), attention and processing speed

Table 2 Test and retest reliability of the BACS among 38 patients with schizophrenia

\begin{tabular}{|c|c|c|c|c|c|c|c|c|}
\hline \multirow[t]{2}{*}{ Subtest of the BACS } & \multicolumn{2}{|c|}{ Test session I } & \multicolumn{2}{|c|}{ Test session 2} & \multicolumn{2}{|c|}{ Correlation } & \multicolumn{2}{|c|}{ Paired $t$-test } \\
\hline & Mean & SD & Mean & SD & ICC & $P$-value & $t$ & $P$-value \\
\hline Verbal memory & 28.47 & 10.54 & 29.63 & 10.39 & 0.735 & $<0.001 *$ & -0.937 & 0.355 \\
\hline Working memory & 16.71 & 4.69 & 17.37 & 5.13 & 0.673 & $<0.00 I^{*}$ & -1.020 & 0.314 \\
\hline Motor speed & 56.95 & 11.23 & 58.21 & 15.68 & 0.699 & $<0.00 I^{*}$ & -0.694 & 0.492 \\
\hline Verbal fluency & 24.95 & 7.30 & 26.08 & 7.64 & 0.592 & $<0.00 I^{*}$ & -1.033 & 0.308 \\
\hline Attention and processing speed & 42.03 & 11.32 & 41.21 & 11.56 & 0.862 & $<0.00 I^{*}$ & 0.837 & 0.408 \\
\hline Executive function & 13.55 & 5.30 & $13.6 \mid$ & 6.25 & 0.720 & $<0.00 I^{*}$ & -0.074 & 0.942 \\
\hline
\end{tabular}

Note: $* P<0.001$.

Abbreviations: BACS, Brief Assessment of Cognition in Schizophrenia; ICC, intraclass correlations; SD, standard deviation. 
Table 3 Correlation matrix of subtests of the BACS in patients with schizophrenia and healthy control subjects

\begin{tabular}{|c|c|c|c|c|c|c|c|}
\hline Subtest of the BACS & VM & WM & MS & VF & APS & EF & CS \\
\hline Verbal memory & - & 0.075 & $0.246 *$ & 0.208 & $0.262^{*}$ & 0.230 & $0.627^{* * *}$ \\
\hline Working memory & $0.617 * * *$ & - & $-0.489 * * *$ & $0.250^{*}$ & 0.137 & 0.045 & $0.324 * *$ \\
\hline Motor speed & $0.337^{* *}$ & 0.112 & - & -0.070 & 0.213 & $0.276^{*}$ & $0.35 I^{* *}$ \\
\hline Verbal fluency & $0.579 * * *$ & $0.52 I^{* * * *}$ & $0.285^{*}$ & - & $0.366 * *$ & 0.132 & $0.583 * * *$ \\
\hline Attention and processing speed & $0.78 I^{* * * *}$ & $0.623 * * *$ & $0.433 * * *$ & $0.498 * * *$ & - & $0.390 * *$ & $0.732 * * *$ \\
\hline Executive function & $0.606 * * *$ & $0.601 * * *$ & $0.343 * *$ & $0.35 I^{* *}$ & $0.630 * * *$ & - & $0.645^{* * *}$ \\
\hline Composite score & $0.860 * * *$ & $0.713 * * *$ & $0.566 * * *$ & $0.67 \mid * * *$ & $0.869 * * *$ & $0.830 * * *$ & - \\
\hline
\end{tabular}

Notes: Correlations in patients with schizophrenia below the diagonal; correlations in healthy control subjects above the diagonal; $* P<0.05$, $* * P<0.01, * * * P<0.00$ I.

Abbreviations: APS, attention and processing speed; BACS, Brief Assessment of Cognition in Schizophrenia; CS, composite score; EF, executive function; MS, motor speed; VF, verbal fluency; VM, verbal memory; WM, working memory.

(AUC: $0.90, P<0.001$ ), working memory (AUC: 0.88, $P<0.001$ ), verbal memory (AUC: $0.84, P<0.001$ ), verbal fluency (AUC: $0.84, P<0.001$ ), and executive function (AUC: $0.77, P<0.001$ ), respectively (Table 4). The ROC output indicated that a composite $Z$-score of -0.71 had the best discriminant validity, with a sensitivity of 0.91 and a specificity of 0.89 .

Among patients with schizophrenia, no significant differences in BACS composite scores were observed between different antipsychotic users $(P=0.066)$. The BACS composite scores were not significantly correlated to the DDD of antipsychotics $(r=-0.07, P=0.561)$.

\section{Discussion}

We found that all BACS subtests in patients with schizophrenia have good test-retest reliability, represented by

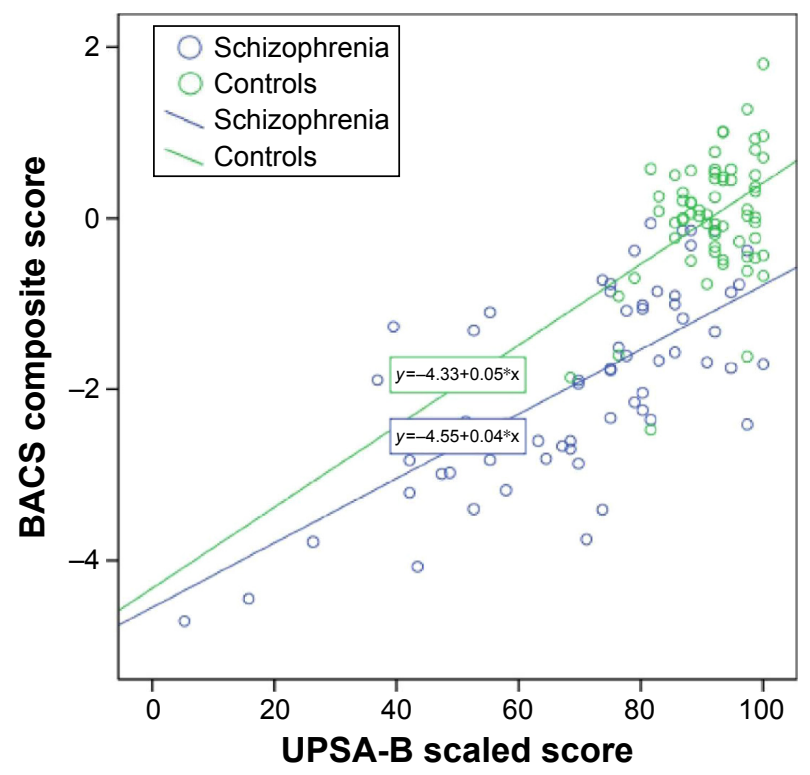

Figure I Scatter plot of the composite scores of the BACS and the UPSA-B scaled scores for patients with schizophrenia and healthy controls (patients: $r=0.70$, $P<0.00$ I; healthy controls: $r=0.44, P<0.00$ I).

Abbreviations: BACS, Brief Assessment of Cognition in Schizophrenia; UPSA-B, UCSD Performance-Based Skills Assessment, brief version.
ICC. The practice effects in all BACS domains estimated by paired samples $t$-test were nonsignificant. Consistent with previous findings regarding the validation of BACS in other language versions, ${ }^{16,17,21,23}$ we suggest that the Chinese version of the BACS is a reliable assessment tool for understanding the cognitive function of patients with schizophrenia. Keefe et $\mathrm{al}^{16}$ suggested that forms of the list learning test and Tower of London test need to be changed to retest patients and assess changes in their cognitive abilities independent of practice effects (such as memorization resulting from repeated test taking). Therefore, all patients were given an alternative form of the list learning test and Tower of London test at the time of retesting in our study. Notably, the category instances test (verbal fluency) differs between the English and the Chinese versions of the BACS. There are two alternative forms of the category instances test

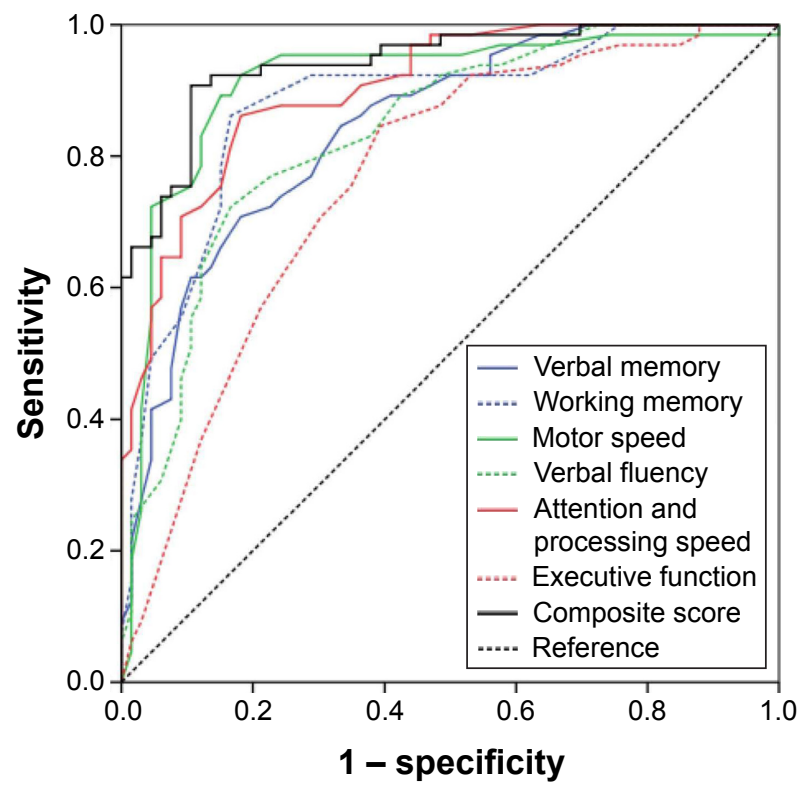

Figure 2 The receiver operating characteristic analysis for subtests of the Chinese version of the BACS in discriminating patients with schizophrenia and healthy controls.

Abbreviation: BACS, Brief Assessment of Cognition in Schizophrenia. 
Table 4 The discrimination validity of cognitive performance measured by the BACS in patients with schizophrenia and healthy controls, using the ROC analyses

\begin{tabular}{lllll}
\hline Subtest of the BACS & AUC & $\mathbf{9 5 \%} \mathbf{C l}$ & Standard error & P-value \\
\hline Verbal memory & 0.844 & $0.778-0.909$ & 0.033 & $<0.00 I^{*}$ \\
Working memory & 0.877 & $0.816-0.938$ & 0.031 & $<0.00 I^{*}$ \\
Motor speed & 0.912 & $0.857-0.967$ & 0.028 & $<0.00 I^{*}$ \\
Verbal fluency & 0.836 & $0.768-0.904$ & 0.035 & $<0.00 I^{*}$ \\
Attention and processing speed & 0.903 & $0.853-0.952$ & 0.025 & $<0.00 I^{*}$ \\
Executive function & 0.765 & $0.683-0.847$ & 0.042 & $<0.00 I^{*}$ \\
Composite score & 0.942 & $0.904-0.980$ & 0.019 & $<0.00 I^{*}$ \\
\hline
\end{tabular}

Note: $* P<0.001$.

Abbreviations: AUC, area under curve; BACS, Brief Assessment of Cognition in Schizophrenia; Cl, confidence interval; ROC, receiver operating characteristic.

(naming supermarket items or tools) in the English BACS and only one form (naming animals) in the Chinese BACS. Therefore, no alternative form of the verbal fluency test could be used in the current study.

The PCA performed in our study (similar to previous studies of the Spanish, ${ }^{20}$ Persian, ${ }^{22}$ and Portuguese versions of the BACS $)^{23}$ revealed that one factor accounted for $60.9 \%$ of the total variance. Nevertheless, a significantly better model fit was also associated with a three-factor solution in the original English BACS ${ }^{16}$ and Japanese BACS, ${ }^{17}$ and a two-factor solution in the French BACS. ${ }^{18}$ The components extracted by PCA might be different between studies because of discrepancies in sample characteristics, sample size, language or culture, and clinical features. ${ }^{31,32}$ Nevertheless, all aforementioned studies consistently indicated that a single factor could explain a large amount of the variance (general cognitive function). Taken together, these findings indicate that the BACS can be used to assess specific cognitive domains, as well as general cognitive function in schizophrenia.

In agreement with the previous validation studies, ${ }^{16,20-22}$ our study showed strong correlation between BACS composite scores and all BACS subscales, in both patients and controls and between BACS composite scores and UPSA-B scores. Previous researchers also found strong correlation between the BACS composite score and standard neurocognitive assessments other than the BACS. ${ }^{16-18,20,22,23,33}$ These findings indicate that the BACS has satisfactory intercorrelations and concurrent validity. However, the magnitude of our correlation coefficients between BACS subtests (Table 3), as well as between BACS and UPSA-B scores (Figure 1), was lower in healthy controls than in patients with schizophrenia. These results may be explained by the global nature of cognitive decline in patients but not healthy controls, giving rise to strong correlation among specific domains of cognitive function. In addition, the UPSA-B score is a measure of basic real-world functioning in patients with mental illnesses, ${ }^{29}$ and an obvious ceiling effect may be present in healthy individuals without cognitive impairments.

The ROC analysis (Figure 2) showed the high sensitivity and specificity of the BACS composite score to distinguish patients from controls ( $\mathrm{AUC}=0.94$ ). This finding is in line with German BACS ${ }^{21}$ and Persian BACS findings. ${ }^{22}$ This result suggests that the Chinese BACS has good discriminant validity for distinguishing patients from controls based on cognitive function. Among the BACS subtests, motor speed and attention and processing speed also individually had a high discriminant validity (AUC $>0.9$ ), while executive function, measured by the Tower of London test, had a relatively low discriminant validity $(\mathrm{AUC}=0.77)$. Likewise, Keefe et $\mathrm{a}^{16}$ previously found that discriminant validity between patients and controls was smaller for executive function than for other BACS subtests. This implies that the Tower of London test is less sensitive to cognitive deficit detection in schizophrenia. Furthermore, cognitive function has been associated with clinical symptoms or prescription of psychotropic drugs in patients with schizophrenia. ${ }^{34-36}$ The large deficits in motor speed and attention of schizophrenia patients may possibly be due to disease course or antipsychotic drug therapy.

This study has several limitations: first, the sample size in this study was small. Further study with a larger sample size is warranted to examine the reliability and validity of the BACS across different clinical groups (eg, paranoid type vs disorganized type; first-episode psychosis vs chronic schizophrenia). Second, only patients with schizophrenia, but not healthy control subjects, were given BACS retests. In addition, the second form of the list learning test was constantly used for BACS retesting. Therefore, we could not determine ICCs and practice effects in healthy individuals, nor compare test-retest reliability across different versions of the list learning test. Third, this study used only 
one cognitive test (UPSA-B) as the validity criterion for the BACS. A standard battery with less ceiling effect is needed to certify the concurrent validity of the BACS in future study. Finally, Chinese contains a group of related language varieties, and the participants in this study were tested in Mandarin (the most widely used dialect in Taiwan and People's Republic of China). It remains uncertain whether the findings in this study could be generalized to population who speak other dialects.

\section{Conclusion}

In summary, the Chinese version of the BACS provides satisfactory psychometric properties, including high test-retest reliability, high internal consistency, acceptable concurrent validity, and good overall and domain-specific discriminant validity. We suggest that the Chinese BACS is a reliable and practical tool for assessing cognitive function in patients with schizophrenia.

\section{Acknowledgments}

This study was funded by Chang Gung Memorial Hospital, Taiwan (CMRPG8C1051, CMRPG8C1291, and CMRPG8E1351). The funding sources had no involvement in the study design, collection, analysis and interpretation of data, writing of the report, or the decision to submit the article for publication. The authors express their deepest gratitude to Professor Richard SE Keefe for granting permission to use the Chinese version of the BACS and Miss Joanne Lo for assisting in participant recruitment, and thank all of the individuals who participated in this study.

\section{Disclosure}

The authors report no conflicts of interest in this work.

\section{References}

1. Palmer EC, Gilleen J, David AS. The relationship between cognitive insight and depression in psychosis and schizophrenia: a review and meta-analysis. Schizophr Res. 2015;166(1-3):261-268.

2. Kitchen H, Rofail D, Heron L, Sacco P. Cognitive impairment associated with schizophrenia: a review of the humanistic burden. Adv Ther. 2012; 29(2):148-162.

3. Bortolato B, Miskowiak KW, Kohler CA, Vieta E, Carvalho AF. Cognitive dysfunction in bipolar disorder and schizophrenia: a systematic review of meta-analyses. Neuropsychiatr Dis Treat. 2015;11: 3111-3125.

4. Barch DM, Ceaser A. Cognition in schizophrenia: core psychological and neural mechanisms. Trends Cogn Sci. 2012;16(1):27-34.

5. Hoe M, Nakagami E, Green MF, Brekke JS. The causal relationships between neurocognition, social cognition and functional outcome over time in schizophrenia: a latent difference score approach. Psychol Med. 2012;42(11):2287-2299.

6. Kalkstein S, Hurford I, Gur RC. Neurocognition in schizophrenia. Curr Top Behav Neurosci. 2010;4:373-390.
7. Schaefer J, Giangrande E, Weinberger DR, Dickinson D. The global cognitive impairment in schizophrenia: consistent over decades and around the world. Schizophr Res. 2013;150(1):42-50.

8. Keefe RS, Harvey PD. Cognitive impairment in schizophrenia. Handb Exp Pharmacol. 2012;213:11-37.

9. Lin CY, Tsai GE, Lane HY. Assessing and treating cognitive impairment in schizophrenia: current and future. Curr Pharm Des. 2014;20(32): $5127-5138$.

10. Bakkour N, Samp J, Akhras K, et al. Systematic review of appropriate cognitive assessment instruments used in clinical trials of schizophrenia, major depressive disorder and bipolar disorder. Psychiatry Res. 2014;216(3):291-302.

11. Buchanan RW, Davis M, Goff D, et al. A summary of the FDA-NIMHMATRICS workshop on clinical trial design for neurocognitive drugs for schizophrenia. Schizophr Bull. 2005;31(1):5-19.

12. Keefe RS, Fox KH, Harvey PD, Cucchiaro J, Siu C, Loebel A. Characteristics of the MATRICS Consensus Cognitive Battery in a 29-site antipsychotic schizophrenia clinical trial. Schizophr Res. 2011;125(2-3): 161-168.

13. Keefe RS, Harvey PD, Goldberg TE, et al. Norms and standardization of the Brief Assessment of Cognition in Schizophrenia (BACS). Schizophr Res. 2008;102(1-3):108-115.

14. Keefe RS, Poe M, Walker TM, Harvey PD. The relationship of the Brief Assessment of Cognition in Schizophrenia (BACS) to functional capacity and real-world functional outcome. J Clin Exp Neuropsychol. 2006;28(2):260-269.

15. Keefe RS, Poe M, Walker TM, Kang JW, Harvey PD. The Schizophrenia Cognition Rating Scale: an interview-based assessment and its relationship to cognition, real-world functioning, and functional capacity. Am J Psychiatry. 2006;163(3):426-432.

16. Keefe RS, Goldberg TE, Harvey PD, Gold JM, Poe MP, Coughenour L. The Brief Assessment of Cognition in Schizophrenia: reliability, sensitivity, and comparison with a standard neurocognitive battery. Schizophr Res. 2004;68(2-3):283-297.

17. Kaneda Y, Sumiyoshi T, Keefe R, Ishimoto Y, Numata S, Ohmori T. Brief assessment of cognition in schizophrenia: validation of the Japanese version. Psychiatry Clin Neurosci. 2007;61(6):602-609.

18. Bralet MC, Falissard B, Neveu X, Lucas-Ross M, Eskenazi AM, Keefe RS. Validation of the French version of the BACS (the brief assessment of cognition in schizophrenia) among 50 French schizophrenic patients. Eur Psychiatry. 2007;22(6):365-370.

19. Anselmetti S, Poletti S, Ermoli E, et al. The Brief Assessment of Cognition in Schizophrenia. Normative data for the Italian population. Neurol Sci. 2008;29(2):85-92.

20. Segarra N, Bernardo M, Gutierrez F, et al. Spanish validation of the Brief Assessment in Cognition in Schizophrenia (BACS) in patients with schizophrenia and healthy controls. Eur Psychiatry. 2011; 26(2):69-73

21. Sachs G, Winklbaur B, Jagsch R, Keefe RS. Validation of the German version of the Brief Assessment of Cognition in Schizophrenia (BACS) - preliminary results. Eur Psychiatry. 2011;26(2):74-77.

22. Mazhari S, Parvaresh N, Eslami Shahrbabaki M, Sadeghi MM, Nakhaee N, Keefe RS. Validation of the Persian version of the Brief Assessment Of Cognition in Schizophrenia in patients with schizophrenia and healthy controls. Psychiatry Clin Neurosci. 2014;68(2): $160-166$.

23. Araujo GE, Resende CB, Cardoso AC, Teixeira AL, Keefe RS, Salgado JV. Validity and reliability of the Brazilian Portuguese version of the BACS (Brief Assessment of Cognition in Schizophrenia). Clinics (Sao Paulo). 2015;70(4):278-282.

24. American Psychiatric Association. Diagnostic and Statistical Manual of Mental disorders (DSM-IV-TR). Washington, DC: American Psychiatric Association; 2000.

25. Sheehan DV, Lecrubier Y, Sheehan KH, et al. The Mini-International Neuropsychiatric Interview (M.I.N.I.): the development and validation of a structured diagnostic psychiatric interview for DSM-IV and ICD-10. J Clin Psychiatry. 1998;59(Suppl 20):22-33; quiz 34-57. 
26. Hochberger WC, Hill SK, Nelson CL, et al. Unitary construct of generalized cognitive ability underlying BACS performance across psychotic disorders and in their first-degree relatives. Schizophr Res. 2016;170(1):156-161.

27. WHO Collaborating Centre for Drug Statistics Methodology. ATC/DDD Index. 2016. Available from: http://www.whocc.no/atc_ddd_index/. Accessed August 25, 2016.

28. Patterson TL, Goldman S, McKibbin CL, Hughs T, Jeste DV. UCSD Performance-Based Skills Assessment: development of a new measure of everyday functioning for severely mentally ill adults. Schizophr Bull. 2001;27(2):235-245.

29. Mausbach BT, Harvey PD, Goldman SR, Jeste DV, Patterson TL. Development of a brief scale of everyday functioning in persons with serious mental illness. Schizophr Bull. 2007;33(6):1364-1372.

30. McIntosh BJ, Zhang XY, Kosten T, et al. Performance-based assessment of functional skills in severe mental illness: results of a large-scale study in China. J Psychiatr Res. 2011;45(8):1089-1094.

31. Kaneda A, Katagai T, Yasui-Furukori N. Comparing the influences of age and disease on the Brief Assessment of Cognition in Schizophrenia in Japanese patients with schizophrenia. Neuropsychiatr Dis Treat. 2013; 9:1203-1208
32. Norbury CF, Sparks A. Difference or disorder? Cultural issues in understanding neurodevelopmental disorders. Dev Psychol. 2013;49(1): 45-58.

33. Yoshida T, Suga M, Arima K, et al. Criterion and construct validity of the CogState Schizophrenia Battery in Japanese patients with schizophrenia. PLoS One. 2011;6(5):e20469.

34. Kishi T, Moriwaki M, Kawashima K, et al. Investigation of clinical factors influencing cognitive function in Japanese schizophrenia. Neurosci Res. 2010;66(4):340-344.

35. Hori H, Yoshimura R, Katsuki A, et al. The cognitive profile of aripiprazole differs from that of other atypical antipsychotics in schizophrenia patients. J Psychiatr Res. 2012;46(6):757-761.

36. Lin $\mathrm{CH}$, Huang $\mathrm{CL}$, Chang $\mathrm{YC}$, et al. Clinical symptoms, mainly negative symptoms, mediate the influence of neurocognition and social cognition on functional outcome of schizophrenia. Schizophr Res. 2013;146(1-3):231-237.

\section{Publish your work in this journal}

Neuropsychiatric Disease and Treatment is an international, peerreviewed journal of clinical therapeutics and pharmacology focusing on concise rapid reporting of clinical or pre-clinical studies on a range of neuropsychiatric and neurological disorders. This journal is indexed on PubMed Central, the 'PsycINFO' database and CAS, and is the official journal of The International Neuropsychiatric Association (INA). The manuscript management system is completely online and includes a very quick and fair peer-review system, which is all easy to use. Visit http://www.dovepress.com/testimonials.php to read real quotes from published authors. 